Boise State University

ScholarWorks

4-2020

\title{
Relevance of Individual and Environmental Drivers of Movement of Golden Eagles
}

Maitreyi Sur

Boise State University

\section{Publication Information}

Sur, Maitreyi; Duerr, Adam E.; Bell, Douglas A.; Fisher, Robert N.; Tracey, Jeff A.; Bloom, Peter H.; . . and Katzner, Todd E. (2020). "Relevance of Individual and Environmental Drivers of Movement of Golden Eagles". IBIS, 162(2), 381-399. https://dx.doi.org/10.1111/ibi.12766

This document was originally published in IBIS by Wiley-Blackwell on behalf of the U.S. Government. Copyright restrictions may apply. doi: 10.1111/ibi.12766

This article is a U.S. Government work and is in the public domain in the USA. 


\title{
Relevance of individual and environmental drivers of movement of Golden Eagles
}

\author{
MAITREYI SUR $* 1$ (iD ADAM E. DUERR, ${ }^{2}$ (D) DOUGLAS A. BELL, ${ }^{3}$ ROBERT N. FISHER, ${ }^{4}$ \\ JEFF A. TRACEY, ${ }^{4}$ (D) PETER H. BLOOM, ${ }^{2}$ TRICIA A. MILLER $^{5}$ \& TODD E. KATZNER ${ }^{6}$ \\ ${ }^{1}$ Boise State University, 1910 University Drive, Boise, ID, 83725, USA \\ ${ }^{2}$ Bloom Research Inc., Los Angeles, CA, 90019, USA \\ ${ }^{3}$ East Bay Regional Park District, Oakland, CA, 94605, USA \\ ${ }^{4}$ U.S. Geological Survey, Western Ecological Research Center, San Diego, CA,9210, USA \\ ${ }^{5}$ Conservation Science Global, Inc., 303 West Drive, West Cape May, NJ, 08204, USA \\ ${ }^{6}$ U.S. Geological Survey, Forest and Rangeland Ecosystem Science Center, 970 Lusk Street, Boise, \\ ID, 83706, USA
}

An animal's movement is expected to be governed by an interplay between goals determined by its internal state and energetic costs associated with navigating through the external environment. Understanding this ecological process is challenging when an animal moves in two dimensions and even more difficult for birds that move in a third dimension. To understand the dynamic interaction between the internal state of an animal and the variable external environment, we evaluated hypotheses explaining association of different covariates of movement and the trade-offs birds face as they make behavioural decisions in a fluctuating landscape. We used 870000 GPS telemetry data points collected from 68 Golden Eagles Aquila chrysaetos to test demographic, diel, topographic and meteorological hypotheses to determine (1) the probability that these birds would be in motion and (2), once in motion, their flight speed. A complex and sometimes interacting set of potential internal and external factors determined movement behaviour. There was good evidence that reproductive state, manifested as age, sex and seasonal effects, had a significant influence on the probability of being in motion and, to a lesser extent, on speed of motion. Likewise, movement responses to the external environment were often unexpectedly strong. These responses, to northness of slope, strength of orographic updraft and intensity of solar radiation, were regionally and temporally variable. In contrast to previous work showing the role of a single environmental factor in determining movement decisions, our analyses support the hypothesis that multiple factors simultaneously interact to influence animal movement. In particular they highlighted how movement is influenced by the interaction between the individual's internal reproductive state and the external environment, and that, of the environmental factors, topographic influences are often more relevant than meteorological influences in determining patterns of flight behaviour. Further disentangling of how these internal and externals states jointly affect movement will provide additional insights into the energetic costs of movement and benefits associated with achieving process-driven goals.

Keywords: California, demography, Golden Eagle, GPS telemetry, meteorology, movement ecology.

*Corresponding author.

Email: maitreyisur@boisestate.edu 
Movement is fundamental to animal ecology and plays a critical role in many small- and large-scale processes. Animals vary movement to achieve a complex set of goals that, once obtained, are expected to contribute to fitness. As such, hypotheses explaining animal movement have been linked to territorial defence (Graf et al. 2016), den or nest construction (Mainwaring \& Hartley 2013), fulfilling reproductive or food-gathering goals (Weimerskirch et al. 2014), or transiting between daily or seasonally varying ranges (Wittemyer et al. 2008). Although the internal state of animals motivates them to move, the environment the animal experiences is also expected to drive much of the variation in these movements. For example, territories are larger in resource-poor habitats (Smith \& Shugart 1987), movements associated with nest- or den-building and reproduction may occur only when resources are sufficient for breeding (Sergio \& Newton 2003), foraging behaviour will reflect the availability of food and water in the landscape (Wittemyer et al. 2008) and characteristics of migration, and even the decision to migrate, are driven by food availability and migration subsidies (Clobert et al. 2009). To add complexity to this problem, demographic characteristics - age and sex - are expected to influence each of these, with differences in behaviour between males and females and among adults and young animals (Miller et al. 2016). Thus, it is thought that animal movement is ultimately defined by the trade-offs between the environment that determines the energetic cost of movement and the benefits associated with achieving process-driven goals (Halsey 2016).

Understanding how internal and environmental states interact to determine movement is challenging when the animal moves in two dimensions. However, this understanding is even more difficult to achieve when movement occurs in three dimensions. Although all animals respond to their environment when making movement decisions, animals that move in three dimensions have a more complex environmental response because they respond to conditions not only on the ground but also to those in the medium through which they are travelling (i.e. a seascape or an aeroscape; Diehl 2013). For example, movements of marine animals are strongly influenced by abiotic factors such as water temperature, salinity and dissolved oxygen content, which affect the physiology of either the animal or the prey upon which they depend (Hays et al. 2016). Similarly, movement of soaring birds may be constrained by thermal generation (Duerr et al. 2015), topography (Katzner et al. 2012, Pirotta et al. 2018) and the distribution of seasonal winds (Vansteelant et al. 2017).

In the face of internal competition among behavioural goals, energetic costs, age- and sex-specific needs, and environmental constraints, animals face a suite of fundamental trade-offs when making decisions about when and how to move. To understand the interaction of the potential internal state of an animal with its external environment, we evaluated demographic, diel, topographic and meteorological hypotheses explaining the behaviour of soaring birds. We specifically asked which covariates, or combinations of covariates, determine (1) the probability that these birds would chose to be in motion and (2), once in motion, their flight speed. Subsequently, as a single factor was unlikely fully to explain either behaviour, for each behaviour, we evaluated the relative influence of key covariates. By testing these hypotheses among behaviours and age-classes, we gain unique insight into the trade-offs these animals face as they make decisions about movement.

\section{METHODS}

\section{Study species}

Golden Eagles Aquila chrysaetos are large soaring predatory birds with a Holarctic distribution. Some populations are long-distance migrants and others are year-round residents on or near nesting areas. Eagle movements are determined by both their ageand sex-specific goals and the environment they experience (Miller et al. 2016, 2017). For example, adults generally hold breeding territories in a fixed area, but non-territorial adults and pre-adults wander more widely, and males and females have different roles during the nesting cycle. When not moving, Eagles generally perch or roost in trees or on prominent ground features and they can remain in one spot for hours, when hunting, incubating, or in bad weather (Watson 2010). When moving, Eagles generally fly (walking is rare and covers comparatively short distances), and they usually use environmentally generated updrafts for long-distance soaring and gliding (Katzner et al. 2012). Flapping flight is less common and is generally used when moving short distances at low flight altitudes. 


\section{Study site}

We tracked Golden Eagles in and around the state of California, USA. For the purposes of this study, we considered tracking data from ecologically and physiographically distinct provinces called Bird Conservation Regions (BCRs; NABCI 2000).

\section{Telemetry data collection}

To capture Golden Eagles we used bow, cannon or rocket net traps set over carcasses (Bloom et al. 2007) or we hand-captured young birds in the nest. Upon capture, each bird was aged using moult patterns (Jollie 1947, Bloom \& Clark 2001) as sub-adult (nestling and first-year to 4 years) or adult ( $>4$ years). Sex of the birds was determined based on morphology (Bortolotti 1984, Edwards \& Kochert 1986, Watson 2010) and, for a subset, verified by genetic testing $(n=38$; Doyle et al. 2014). Eagles were outfitted with 80-95 g solarpowered GPS/GSM transmitters produced by Cellular Tracking Technologies (Rio Grande, NJ, USA) attached as backpacks using non-abrasive Teflon ribbon harness (Bally Ribbon Mills, Bally, PA, USA; Dunstan 1972, Kenward 1985). The telemetry units were programmed to collect information on GPS locations, altitude, speed, fix quality (2D or 3D fix), horizontal and vertical dilution of precision (HDOP and VDOP) at either 15-min or 30-s intervals. Data were stored on the units and uploaded to the internet at regular intervals through GSM (Global System for Mobile Communications) networks.

\section{Telemetry data processing}

We used a multi-step process to filter out inaccurate and imprecise GPS fixes and to organize our data for analysis. First, we removed 2D fixes and fixes where HDOP or VDOP was $\geq 10$ (D'Eon \& Delparte 2005, Poessel et al. 2016). Secondly, we subsampled all 30-s data to $15-\mathrm{min}$ intervals to standardize fix intervals. Thirdly, we removed the few data points before 04:00 $\mathrm{h}$ and after 19:00 h local time (UTC -8). Finally, we associated Eagle locations with BCRs and a suite of environmental data (see 'Data associations' below for details). We eliminated from consideration all telemetry data from BCRs with $<1000$ GPS points or that were used by fewer than five telemetered birds.
Our analysis focused on two response variables. One described a dichotomous variable of being in motion or not (the probability of being in motion) and the other described behaviour in terms of the hourly speed of motion (Welsh et al. 1996, Fletcher et al. 2005). To calculate these variables, we used the 'Tracking analyst - Track interval to line tool' (ArcGIS 10.3; ESRI, Redland, CA, USA) to convert locations to 'tracks' by joining sequential GPS locations. We then measured the length of each track (in $\mathrm{km}$ ) and the duration between GPS locations considered in a track (in h). We estimated the total distance travelled by Eagles in an hour by summing the lengths of all the tracks between the GPS points nearest to start and end of each nominal hour (e.g. 06:00-07:00 h). Similarly, we estimated total duration of travel in an hour by summing the duration (time between two sequential GPS locations) of all the tracks within an hour. We then calculated hourly speeds from these data (in $\mathrm{km} / \mathrm{h}$ ) by dividing the total distance travelled by Eagles by the total duration (Rus et al. 2017). We classified the bird as either 'moving', when average speed over the entire hour was $\geq 0.05 \mathrm{~km} / \mathrm{h}$, or 'not moving', when average speed was $<0.05 \mathrm{~km} /$ $h$. The rationale for choosing this threshold is given in Figure S1. This binomial variable (moving/not moving) was our first response variable describing the probability of being in motion. We then calculated hourly speed of motion only for the subset of hours in which Eagles were moving. These data became our second response variable.

\section{Data associations}

Many analyses of flight behaviour of birds focus on evaluating response to a single category of environmental variable (e.g. meteorological variables, Sapir et al. 2011, Nagy et al. 2018; or topographic variables, Katzner et al. 2012). We associated each hourly speed measurement with multiple categories of covariates - demographic, topographic, meteorological and diel - which we averaged across all Eagle locations in an hour. The specific covariates we considered were:

\section{Demographic/locational/seasonal (hereafter simply 'demographic')}

Age (sub-adult or adult), assessed at capture and then adjusted for each subsequent year of tracking, and sex (male or female). We described locational information as BCRs (U.S. NABCI Committee 
2000). Our analysis included locations from four BCRs: the Great Basin (BCR 9), Sierra Nevada (BCR 15), Coastal California (BCR 32), and Sonoran and Mojave Desert (BCR 33; Fig. 1). A brief description of each of these BCRs is provided in Data S1; longer descriptions are provided by the North American Bird Conservation Initiative (U.S. NABCI Committee 2000). We also considered month of the year provided by the GPS as a seasonal covariate.

\section{Topographic}

Ground elevation above sea level (m), slope (degrees), aspect (degrees), terrain position index (TPI; Jenness et al. 2013) and terrain ruggedness index (TRI; Riley et al. 1999, Evans et al. 2014) at $30-\mathrm{m}$ resolution. Values of these variables were calculated within ArcGIS 10.3 (ESRI) from the National Elevation Dataset (USGS 2015). To avoid problems associated with analysis of circular statistics, aspect was converted into northness and eastness (Roberts 1986). TPI was converted to categorical variables as either 'canyons', 'steep slopes', 'gentle slopes' or 'ridges' using the Topography Tools for ArcGIS (Jenness et al. 2013, Dilts 2015). TRI, which reflects landscape roughness, was calculated as the square root of the sum of the squared differences between the elevation in a cell and the elevation of its neighbouring cells (Riley et al. 1999, Evans et al. 2014).

\section{Meteorological}

Downward solar radiation (DSR; $\mathrm{W} / \mathrm{m}^{2}$ ), planetary boundary layer height (PBLH; $\mathrm{m})$, surface temperature $\left(\right.$ Temp, ${ }^{\circ} \mathrm{K}$ ), precipitation rate (Precip; kg/ $\mathrm{m}^{2} / \mathrm{s}$ ), barometric pressure (Press in pascals; $\mathrm{Pa}$ ), relative humidity at $2 \mathrm{~m}$ above the ground (Hum; $\%)$, sensible heat flux at the surface (SHF; $\mathrm{W} / \mathrm{m}^{2}$ ), surface lifted index at 500-1000 $\mathrm{mb}$ (SLI; ${ }^{\circ} \mathrm{K}$ ), $u$ wind and $v$-wind $(\mathrm{m} / \mathrm{s})$, and orographic $(\mathrm{m} / \mathrm{s})$ and thermal updraft $(\mathrm{m} / \mathrm{s})$. We chose meteorological variables that we thought would influence movement behaviour of Golden Eagles. For example, Temp, DSR, PBLH, SHF, barometric pressure and SLI are all known to affect the development of thermals in some way, and wind speed and wind direction are known to affect thermal and orographic updrafts (Duerr et al. 2015, Miller et al. 2016, Shamoun-Baranes et al. 2016).

Values of the 10 meteorological parameters were obtained from the Environmental-Data Automated Track Annotation system (Env-DATA;

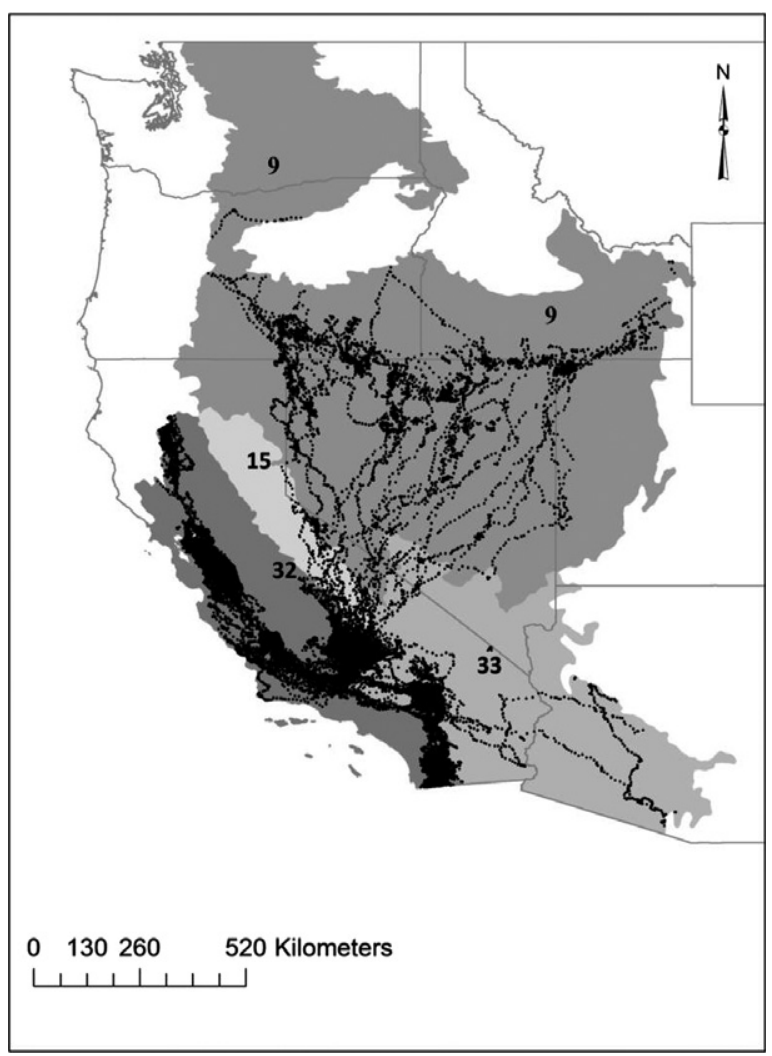

Figure 1. Map of GPS locations (black dots) of 68 Golden Eagles tracked from 2012 to 2016 in western North America. The four Bird Conservation Regions (shaded) are the Great Basin (9), Sierra Nevada (15), Coastal California (32) and Sonoran-Mojave Desert (33).

Dodge et al. 2013) in MOVEBANK (Wikelski \& Kays 2016). Eight of these parameters (all except orographic and thermal updraft) were derived from the NCEP North American regional Reanalysis (NARR) dataset collected at a spatial resolution of $32 \mathrm{~km}$. We calculated wind speed and wind direction from raw wind components ( $u$-wind and $v$-wind) at $10 \mathrm{~m}$ above the ground (Duerr et al. 2015) using the formulae:

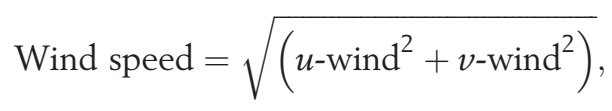

Wind direction $=($ ATAN2 $(v$-wind $/$ wind speed,

$$
\begin{aligned}
& u \text {-wind/wind speed }) * 180 / \pi) \\
& +180 .
\end{aligned}
$$

Orographic and thermal updrafts are derived variables calculated at a spatial resolution of $0.7^{\circ}$ 
and obtained from the Env-DATA system (Dodge et al. 2013) in MOVEBANK (Wikelski \& Kays 2016).

\section{Diel}

Hour of the day provided by the GPS.

\section{Data analysis}

Selecting covariates for inclusion in statistical models We calculated bivariate Pearson correlation coefficients for all possible pairs of averaged, hourly topographic and meteorological covariates (Table S1). When two variables were correlated with each other $(|R|>0.55)$, we only included one of the two in our statistical models (Zuur et al. 2009, Duerr et al. 2015). In these cases, our approach was to retain the single variable that we thought would provide the more logical ecological interpretation (see Table S1 for details on our decision-making process). To evaluate our assessment of the choice of covariates, we also calculated the generalized variance inflation factor (GVIF; Fox \& Monette 1992, Zuur et al. 2010, Tables S2 and S3). That analysis verified that there was no multicollinearity among the variables used in the analysis.

\section{Testing for behavioural responses}

After grouping our explanatory variables into categories, we then used a generalized linear mixed model with a binary response variable and a logit link to understand which variables in each of these categories determined the probability that Eagles would be in motion (our first research question; function: glmer, package: lme4 1.1-15, in program R; Pinheiro et al. 2015, R Core Team 2012). In these models, our response variable was 'moving' or 'not moving'; fixed and random effects in the model are described below.

We subsequently used linear mixed effects models to understand what factors determine the movement behaviour of flying Golden Eagles (our second research question; function: 1mer, package: lme4 1.1-15, in program R). In these models, our response variable was hourly speeds of Eagles. We log-transformed these speed data to conform to the distributional assumptions of our modelling tools. For each model we verified homogeneity of variances by plotting the residuals of the model.

We built separate logistic regression and linear mixed effects model sets for each group of the demographic, topographic and meteorological covariates listed above (Section: Data associations). We included, in all models, random effects for bird ID and for calendar year. Because of the number of Eagle pairs and their geographical distribution, we were unable to collect breeding activity data on all birds and so we use age as an imperfect proxy for sexual maturity. Likewise, because we expected male and female Eagles to behave differently in the breeding season, we included in our demographic models an interaction term describing the relationship between sex and month. To control for temporal autocorrelation in our movement data we included a lagged (lagl) response covariate as a fixed effect (Fieberg \& Ditmer 2012, Van Cleave et al. 2018). We rescaled all continuous meteorological and topographical variables by subtracting the mean and dividing by twice the standard deviation (Gelman 2008). We then used the dredge function of the MuMIN package in $\mathrm{R}$ (Barton 2019) to consider, for each type of explanatory variable, a model set of all possible combinations of sub-models (Doherty et al. 2012). We ranked these sub-models based on the Akaike information criterion (AIC) to identify the model with the most support in the data (Anderson \& Burnham 2002, Anderson 2007). When no single model had $>90 \%$ of model weights, we averaged the top supported models (Anderson \& Burnham 2002). We also calculated variable importance for each fixed effect by summing the AIC weights across all the models in the set where the particular covariate occurred. In the case of the linear mixed effect models, although the global model was fitted using restricted maximum likelihood estimation (REML), when using the dredge function, we fitted the models using maximum likelihood (ML) estimation to allow us to compare the models based on AIC (Zuur et al. 2009). Model averaging was done based on models fitted using ML.

We used mixed effects models to evaluate Eagle response to within-day diel variation. In these models, our two response variables were the probability that a bird would be in motion and hourly speed (both as above), our fixed effects were hour of the day, and we again included random effects for bird ID and calendar year. Again, to account for temporal autocorrelation in our movement data, we included a lagged (lagl) response covariate as a fixed effect. Although we would have preferred to include hour of the day as a fixed effect 
in the demographic, topographic and meteorological models, doing so would have created an unreasonably large number of fixed effects in those models.

It would have been preferable to build a single model set that considered all of these fixed effects together. However, preliminary model runs suggested that given the large number of predictors we considered, such a modelling approach was not computationally feasible. Therefore, to better identify the factors that influence probability of movement and flight speed, we created a model set using all the variables from each of our top demographic, diel, topographic and meteorological models. In this case we compared performance of models by sequentially removing groups of variables (i.e. we 'dredged' groups of variables rather than dredging individual variables). We again ranked models based on AIC to identify those with the most support in the data. These models were again fitted using ML estimation. We also calculated $t$-statistics and $P$-values to identify the significance of all independent variables from the top model. For the logit models, the test statistics were provided as a model summary within the lme4 package itself. For the linear mixed effect model using hourly speed, we used Satterthwaite's method in the lmerTest package in $\mathrm{R}$ to calculate test statistics (Giesbrecht \& Burns 1985, Kuznetsova et al. 2017). We also calculated type III test results for fixed effects; these provide insight into the relative weight and importance of the different covariates in the model (Duerr et al. 2015, Katzner et al. 2015).

\section{RESULTS}

During 2012-2016, we tracked movements of 93 Golden Eagles (40 females and 53 males) captured in California (Table S4). The telemetry devices collected a total of 2875265 GPS locations. After sub-sampling 30-s data and removing poor quality points and points from the bird conservation regions that did not meet our sample size criteria, we retained 872652 locations (Fig. 1). From these locations we calculated 248564 daytime hourly speeds from 68 Golden Eagles.

We interpreted 190895 of those hourly speeds as indicative of moving Eagles (hourly speed $>0.05 \mathrm{~km} / \mathrm{h}$ ) and 57669 as indicative of stationary Eagles. When the birds were moving, the grand mean of their average hourly speed was $3.21 \mathrm{~km} / \mathrm{h}$, with a maximum speed during any $1-\mathrm{h}$ period of $87.31 \mathrm{~km} / \mathrm{h}$. Eagles generally had higher hourly speeds between 11:00 and 14:00 h, with maximum average hourly speed (grand mean $\pm \mathrm{se})$ at $12: 00 \mathrm{~h} \quad(5.19 \pm 0.29 \mathrm{~km} / \mathrm{h}$; range $=0.05-71.47 \mathrm{~km} / \mathrm{h}$ ).

\section{Probability of moving}

All of the demographic variables were strong predictors of the probability of an Eagle being in motion and, in this model set, the full model had 96\% of the model weights (Table 1). This model suggests that adult Eagles were slightly less likely to move compared with sub-adults (Fig. 2a). There was also a strong regional association, such that Eagles were much less likely to be in motion in the Great Basin BCR (Fig. 2b). We also detected an intuitive and strong effect of an interaction between sex and month (Fig. 2c) such that, although both sexes were less likely to be in motion during the nesting season, that effect was especially strong for females. Month had a strong effect on Eagle movements, with a relatively higher probability of birds being in motion in spring and autumn and lower probability of movement in summer and winter.

The probability of an Eagle being in motion was also strongly associated with all topographic variables we considered (Table 1, Fig. 3). In this model set, the full model had model weight $\geq 0.99$. This model suggested that when on more north- and east-facing slopes, Eagles had relatively lower probabilities of being in motion than when on south- and west-facing slopes (Fig. a,b). Likewise, as topographic roughness increased, Eagles were more likely to be in motion (Fig. 3c). Finally, Eagles were most likely to be in motion over steep slopes and less likely to be in motion over canyons, ridges and gentle slopes (Fig. 3d).

Meteorological variables also had strong associations with the probability of an Eagle being in motion (Table 1, Fig. 4). In this model set, the full model had $64 \%$ of model weights and the top four models $99 \%$ of model weights (Table 1). The only difference between the top model and the next three models was that those subsequent models excluded fixed effects describing moisture - relative humidity, precipitation rate or both. Precipitation rate also had the lowest relative variable importance among the nine meteorological 
Table 1. Top five models (ranked by $\triangle A I C$ ) in model sets describing factors affecting the decision to move by Golden Eagles in California, 2012-2016.

\begin{tabular}{|c|c|c|c|c|}
\hline Model type & Model set & AICc & $\triangle \mathrm{AIC}$ & $w_{\mathrm{i}}$ \\
\hline \multirow[t]{5}{*}{ Demographic } & Age + BCR + Month + Sex + Month*Sex + lag & 245957.10 & 0.00 & 0.96 \\
\hline & BCR + Month + Sex + Month*Sex + lag & 245964.42 & 7.32 & 0.02 \\
\hline & Age + BCR + Month + Sex + lag & 245965.39 & 8.29 & 0.02 \\
\hline & Age + BCR + Month + lag & 245968.96 & 11.86 & 0.00 \\
\hline & BCR + Month + Sex + lag & 245972.65 & 15.55 & 0.00 \\
\hline \multirow[t]{5}{*}{ Topographic } & Eastness + Northness + TPI + TRI + lag & 238172.20 & 0.00 & $\geq 0.99$ \\
\hline & Eastness + Northness + TPI + lag & 238194.38 & 22.18 & 0.00 \\
\hline & Northness + TPI + TRI + lag & 238357.70 & 185.50 & 0.00 \\
\hline & Northness + TPI + lag & 238370.49 & 198.29 & 0.00 \\
\hline & Eastness + Northness + TRI + lag & 238474.32 & 302.12 & 0.00 \\
\hline \multirow[t]{5}{*}{ Meteorological } & $\begin{array}{l}\text { DSR + Humid + Orographic + PBLH + Precip + Press } \\
+ \text { SLI + Wind speed + Wind direction + lag }\end{array}$ & 241348.00 & 0.00 & 0.64 \\
\hline & $\begin{array}{l}\text { DSR + Humid + Orographic + PBLH + Press } \\
+ \text { SLI + Wind speed + Wind direction + lag }\end{array}$ & 241349.22 & 1.22 & 0.35 \\
\hline & $\begin{array}{l}\text { DSR + Orographic + PBLH + Press } \\
+ \text { SLI + Wind speed + Wind direction + lag }\end{array}$ & 241359.08 & 11.08 & 0.00 \\
\hline & $\begin{array}{l}\text { DSR + Orographic + PBLH + Precip } \\
+ \text { Press + SLI + Wind speed + Wind } \\
\text { direction + lag }\end{array}$ & 241359.94 & 11.94 & 0.00 \\
\hline & $\begin{array}{l}\text { DSR + Humid + Orographic + PBLH + Precip } \\
+ \text { SLI + Wind speed + Wind direction + lag }\end{array}$ & 241361.76 & 13.76 & 0.00 \\
\hline
\end{tabular}

We used logistic regression to evaluate what factors determine the probability that these birds would be in motion with demographic, topographic and meteorological variables as fixed effects and bird ID and year as random effects. We also added a lagged-response covariate ('lag') as a fixed effect. Model sets were composed of all possible combinations of all factors within each category
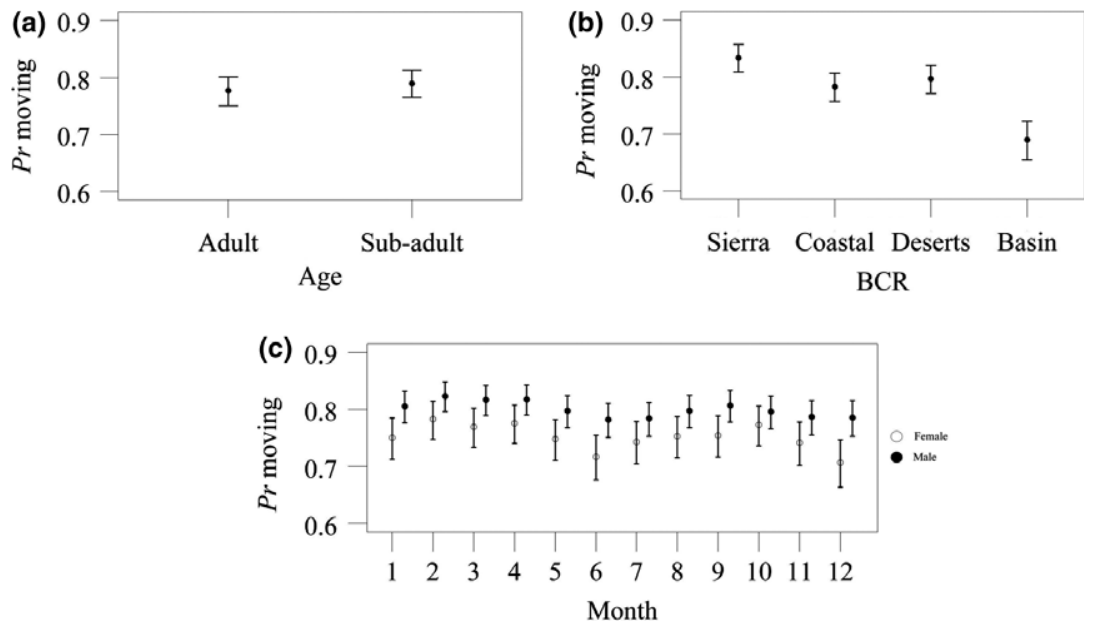

Figure 2. Modelled estimates of the probability that Golden Eagles in California would be in motion, as predicted by demographic parameters (a) age, (b) Bird Conservation Region (BCR), and (c) the interaction of sex and month for female and male Eagles. The four BCRs are the Sierra Nevada (Sierra), Coastal California (Coastal), Sonoran-Mojave Desert (Desert) and Great Basin (Basin). The plots use estimated values from the top demographic model predicting Eagle movement (Table 1).

variables (Table S5). In general, the probability that an Eagle was in flight was positively correlated to downward solar radiation (Fig. 4a), planetary boundary layer height (Fig. 4b), barometric pressure (Fig. 4c), surface lifted index (Fig. 4d), wind speed (Fig. 4e) and orographic updraft (Fig. 4f). However, the probability that an Eagle was in flight was negatively correlated with 

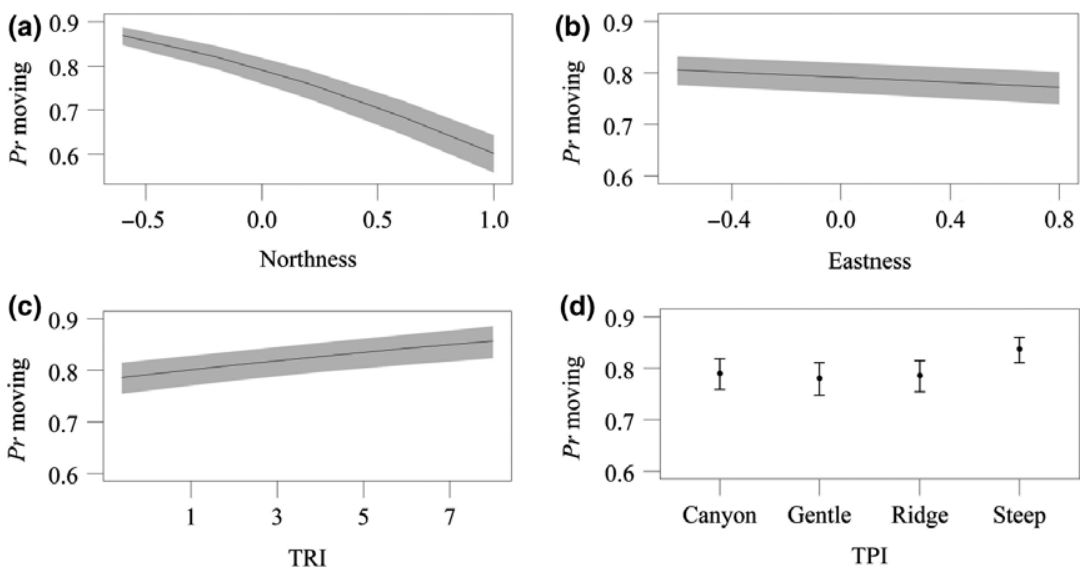

Figure 3. Modelled estimates of the probability that Golden Eagles in California would be in motion, as predicted by the topographic factors (a) northness, (b) eastness, (c) TRI and (d) TPI. The four TPI categories are canyon (canyon), gentle slope (gentle), ridge (ridge) and steep slope (steep). The plot uses estimated values from the top topographic model predicting Eagle movement (Table 1). Variables were rescaled for modelling purposes (see text). Grey bands represent 95\% confidence intervals.
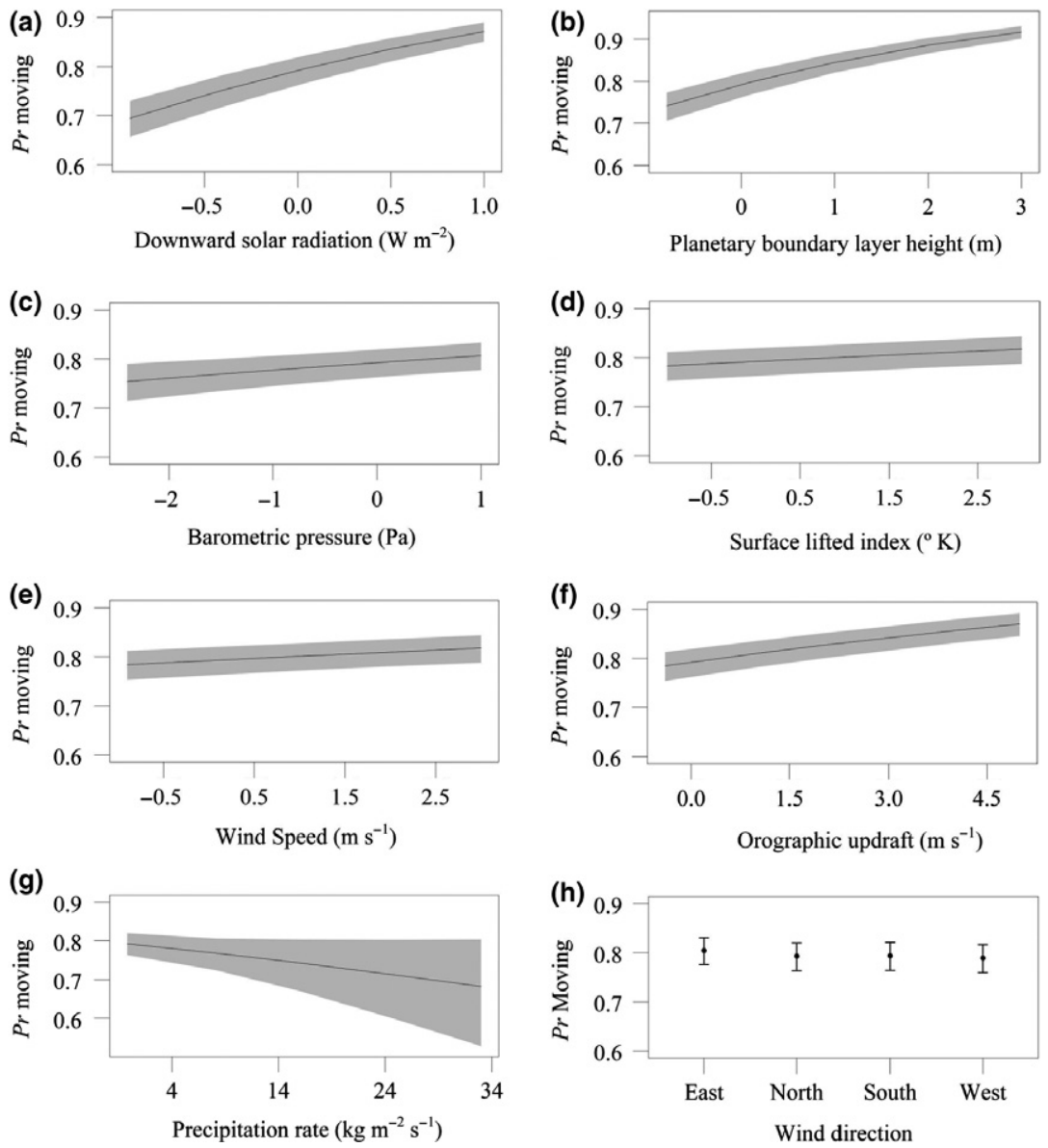

Figure 4. Modelled estimates of the probability that Golden Eagles in California would be in motion, as predicted by meteorological factors (a) downward solar radiation, (b) planetary boundary layer height, (c) barometric pressure, (d) surface lifted index, (e) wind speed, (f) orographic updraft, $(\mathrm{g})$ precipitation rate and $(\mathrm{h})$ wind direction. The plot uses estimated values from the top meteorological model predicting Eagle movement (Table 1). Variables were rescaled for modelling purposes (see text). Grey bands represent $95 \%$ confidence intervals. 

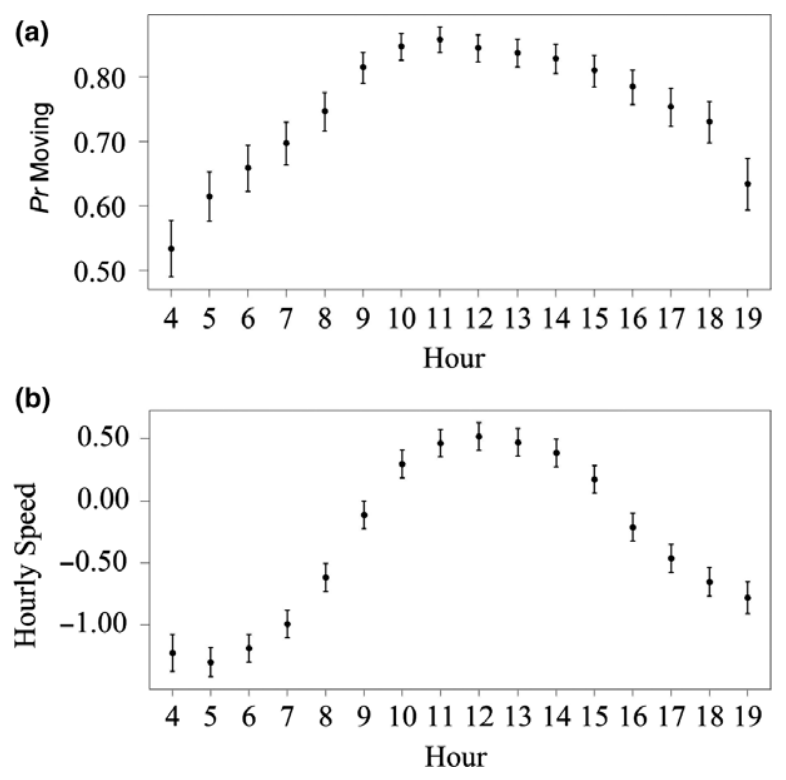

Figure 5. Modelled estimates of the probability that Golden Eagles in California would move, as predicted by hour of the day (a), and estimated values of hourly speed (log-transformed) of Golden Eagles in California as predicted by hour of the day (b). Plots show modelled estimates and 95\% confidence intervals.

precipitation rate (Fig. $4 \mathrm{~g}$ ) and was not strongly influenced by wind direction (Fig. 4h).

There were important diel cycles of an Eagle's probability of being in motion $\left(F_{15}=382.40\right.$, $P<0.05)$. Eagles were most likely to be in motion in the middle of the day, at approximately 11:00 or 12:00 h, and least likely to be in motion early in the mornings and in the evenings (Table S6, Fig. 5a). The probability of being in motion increased at a steeper rate in the morning than it decreased in the afternoon.

The lagged-response covariate was present in all top models of the demographic, topographic, meteorological model sets, and in the diel model. This parameter helped us to account for the effect of the autocorrelation on the probability of being in motion. Its presence in top models suggests that at a given hour, the probability of being in motion was strongly associated with the behaviour of the Eagle in the hour before. This is not surprising, given that the data came from individuals tracked over time and points to the importance of including the lagged-response covariate in this type of analysis.

Combinations of the top models suggested that the probability of being in motion was best explained by the full model with the laggedresponse covariate as well as all the factors from
Table 2. Results of comparison of combinations of the top models from Table 1, describing factors affecting the decision to move by Golden Eagles in California, 2012-2016.

\begin{tabular}{lrrr}
\hline Probability of moving models & AICc & \multicolumn{1}{c}{$\Delta$ AIC } & $w_{\mathrm{i}}$ \\
\hline Topo + Met + Hour + Demo & 231425.20 & 0.00 & 1.00 \\
Topo + Hour + Demo & 231826.96 & 401.76 & 0.00 \\
Topo + Met + Demo & 231999.69 & 574.49 & 0.00 \\
Topo + Met + Hour & 232168.14 & 742.94 & 0.00 \\
Topo + Hour & 232469.31 & 1044.11 & 0.00 \\
Topo + Met & 233849.40 & 2424.20 & 0.00 \\
Met + Hour + Demo & 238930.04 & 7504.84 & 0.00 \\
Met + Demo & 239605.67 & 8180.47 & 0.00 \\
Topo + Demo & 237407.89 & 5982.69 & 0.00 \\
Hour & 240021.61 & 8596.41 & 0.00 \\
Met + Hour & 239496.06 & 8070.86 & 0.00 \\
Met & 241347.97 & 9922.77 & 0.00 \\
Topo & 238172.19 & 6746.99 & 0.00 \\
Hour + Demo & 239476.50 & 8051.30 & 0.00 \\
Demo & 245957.08 & 14531.88 & 0.00 \\
& & &
\end{tabular}

We used logistic regression to evaluate what factors determine the probability that these birds would be in motion with a combination of demographic (Demo), diel (Hour), topographic (Topo) and meteorological (Met) variables from our top models in Table 1 as fixed effects and bird ID and year as random effects. We also added a lagged-response covariate as a fixed effect. We used ascending $\triangle \mathrm{AIC}$ to rank the models

our top demographic, topographic and meteorological models (Table 2). However, by combining these separate models, we were able to rank variables, providing insight into the relative influence of different types of variables on the probability that an Eagle was in motion. Although effect estimates for most variables were non-zero, the relevance of topographic features stood out, primarily because of the high absolute value of the $t$-statistic and

$F$-value for northness and, to a lesser degree, of TPI (Tables S7 and S8). Likewise, there were comparatively strong effects of BCR and hour of the day. Surprisingly, meteorological variables tended to be relatively less influential, although the height of the planetary boundary layer and barometric pressure were the highest ranked of such variables. Finally, age, sex and month of the year were comparatively less influential, and effect estimates for many of the sex by month interactions were not different from zero.

\section{Hourly speed}

Demographic predictors strongly influenced hourly speed of Golden Eagles (Table 3, Fig. 6). In this 
Table 3. Results of the top five models describing factors affecting hourly speeds of Golden Eagles in California, 2012-2016.

\begin{tabular}{|c|c|c|c|c|}
\hline Model type & Model set & AICc & $\triangle \mathrm{AIC}$ & $w_{i}$ \\
\hline \multirow[t]{5}{*}{ Demographic } & Age + BCR + Month + Sex +Sex*Month + lag & 700059.70 & 0.00 & 0.89 \\
\hline & BCR + Month + Sex +Sex*Month + lag & 700063.85 & 4.15 & 0.11 \\
\hline & Age + BCR + Month + lag & 700073.93 & 14.23 & 0.00 \\
\hline & Age + BCR + Month + Sex + lag & 700075.93 & 16.23 & 0.00 \\
\hline & BCR + Month + lag & 700079.58 & 19.88 & 0.00 \\
\hline \multirow[t]{5}{*}{ Topographic } & Eastness + Northness + TPI + TRI + lag & 685622.90 & 0.00 & $\geq 0.99$ \\
\hline & Eastness + Northness + TPI + lag & 686131.03 & 508.13 & 0.00 \\
\hline & Northness + TPI + TRI + lag & 686285.07 & 662.17 & 0.00 \\
\hline & Northness + TPI + lag & 686859.94 & 1237.04 & 0.00 \\
\hline & Eastness + Northness + TRI + lag & 687293.30 & 1670.40 & 0.00 \\
\hline \multirow[t]{5}{*}{ Meteorological } & $\begin{array}{l}\text { DSR + Humid + Orographic + PBLH + Precip } \\
+ \text { Press + SLI + Wind speed + Wind direction + lag }\end{array}$ & 689456.10 & 0.00 & 0.53 \\
\hline & $\begin{array}{l}\text { DSR + Humid + PBLH + Precip + Press + SLI } \\
+ \text { Wind speed + Wind direction + lag }\end{array}$ & 689456.39 & 0.29 & 0.46 \\
\hline & $\begin{array}{l}\text { DSR + Humid + Orographic + PBLH + Precip + SLI } \\
+ \text { Wind speed + Wind direction + lag }\end{array}$ & 689465.84 & 9.74 & 0.00 \\
\hline & $\begin{array}{l}\text { DSR + Humid + PBLH + Precip + SLI + Wind speed } \\
+ \text { Wind direction + lag }\end{array}$ & 689466.69 & 10.59 & 0.00 \\
\hline & $\begin{array}{l}\text { DSR + Humid + PBLH + Precip + Press + SLI } \\
+ \text { Wind direction + lag }\end{array}$ & 689511.03 & 54.93 & 0.00 \\
\hline
\end{tabular}

We used linear mixed effects models with log-transformed hourly speeds of Eagles as the response variable, demographic, diel, topographic and meteorological variables as our fixed effects, and bird ID and year as random effects as described in the text. We also added a lagged-response covariate ('lag') as a fixed effect. We used ascending $\triangle \mathrm{AIC}$ to rank the models.
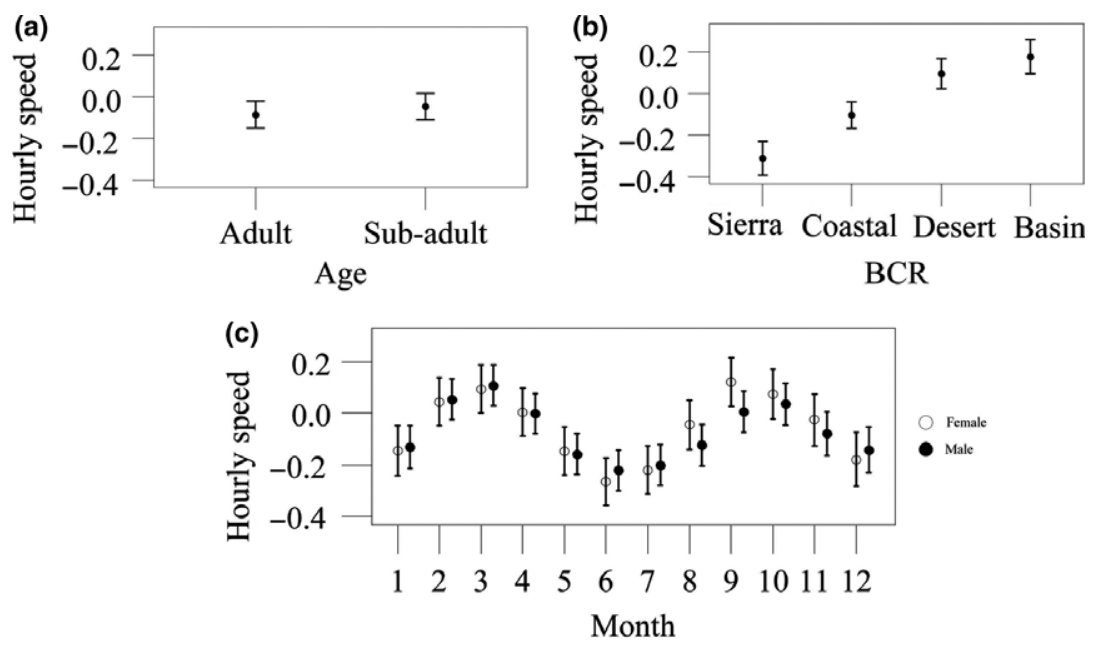

Figure 6. Model estimated values of hourly speed (log-transformed) of Golden Eagles in California, as predicted by demographic variables (a) age, (b) Bird Conservation Region (BCR) and (c) the interaction of sex and month for female and male Eagles. The four BCRs are the Sierra Nevada (Sierra), Coastal California (Coastal), Sonoran-Mojave Desert (Desert) and Great Basin (Basin). The plots use estimated values from the top demographic model predicting hourly speed (Table 3 ).

model set, the top model had $89 \%$ of model weights, and the top two models $99 \%$ of model weights (Tables 3 and S9). The top model suggests that age had an association with hourly speed with sub-adults moving slightly faster than adults (Fig. 6a). We found a strong association of hourly speed with BCR, with lowest hourly speeds in Sierra Nevada and highest in the Great Basin (Fig. 6b). Unlike our previous results we did not detect strong effects of the interaction between sex and month (Fig. 6c), with both sexes showing similar variations in hourly speed with month of 
the year. Month of the year seemed to have a strong effect on hourly speed, with relatively higher speeds during spring and autumn and lower speeds in summer and winter.

Topographic predictors also had strong associations with movement behaviour of Eagles (Table 3, Fig. 7). In this case, the full model had model weight $\geq 0.99$. The model suggested that Eagles moved faster over south- and west-facing slopes than on north- or east-facing slopes (Fig. 7a). Although Eagles were more likely to be in motion in rougher terrains (Fig. 3c), hourly speed actually decreased with increased topographic roughness (Fig. 7c). The model also suggested that Golden Eagles moved fastest over steep slopes and ridges (Fig. 7d).

Meteorological predictors were also associated with hourly speed of Eagles (Table 3, Fig. 8). In this model set, the top model had $53 \%$ of model weights while the second model had $46 \%$ of model weights. The two models differed by inclusion or exclusion of orographic updraft, the fixed effect that had the lowest variable importance (Table S10). Hourly speed of Eagles was positively associated with meteorological variables conducive to the formation of thermals, including downward solar radiation (Fig. 8a), planetary boundary layer height (Fig. 8b), surface lifted index (Fig. 8c) and relative humidity (Fig. 8d). However, although flight speed was slightly positively related to wind speed (Fig. 8e) and orographic updraft (Fig. 8f), it was strongly negatively related to precipitation rate
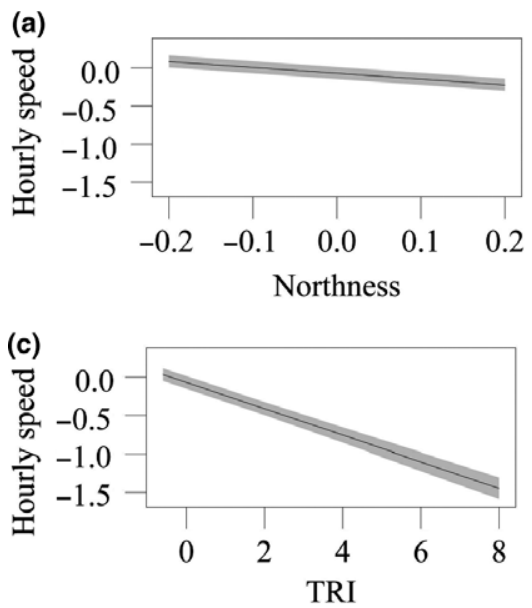

(Fig. 8g). Finally, hourly speed of Eagles was, to a small degree, more influenced by winds from the east, north and south than by winds from the west (Fig. 8h).

We found diel trends in the hourly speed of eagles $\left(F_{15}=1679.5, P<0.05\right.$; Table S11). This analysis suggested that hourly speed varied significantly throughout the day with lowest speeds in the early morning and late evening and highest speeds in the afternoon between 11:00 and 14:00 h (Fig. 5b).

Finally, the lagged-response covariate was again present in all top models. As before, this suggests that the speed of the bird in any given hour was strongly dependent on its speed in the hour before. This observation is consistent with the temporal patterns we observed in this parameter (Fig. 5b).

Combinations of the top models again suggested that hourly speed of Eagles was best explained by all the factors from our top demographic, topographic, meteorological and diel models, as well as the lagged-response covariate (Table 4). These models also provided insight into the relative influence of different types of parameters on Golden Eagle flight speed. The influence of topographic features also stood out in this model and the $t$-statistic and $F$-value of the topographic variables northness, TPI, eastness and TRI had relatively high absolute values (Tables S12 and S13). In contrast to the analysis of the probability of being in motion, there were comparatively strong effects of
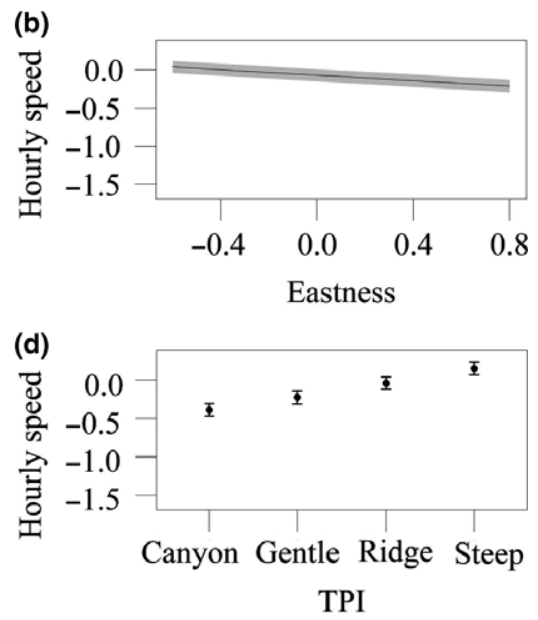

Figure 7. Model estimated values of hourly speed (log-transformed) of Golden Eagles in California, as predicted by topographical variables (a) northness, (b) eastness, (c) TRI and (d) TPI. The four TPIs are canyon (canyon), gentle slope (gentle), ridge (ridge) and steep slope (steep). The plot uses estimated values from the top topographic model predicting hourly speed (Table 3 ). The variables were rescaled for modelling purposes (see text). Grey bands represent $95 \%$ confidence intervals. 


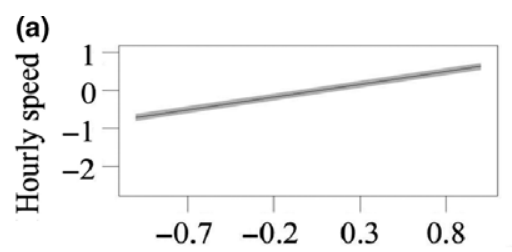

Downward solar radiation $\left(\mathrm{W} \mathrm{m}^{-2}\right)$

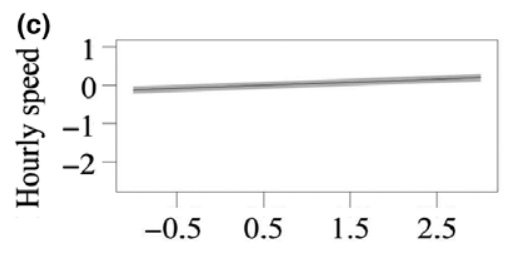

Surface lifted index $\left({ }^{\circ} \mathrm{K}\right)$
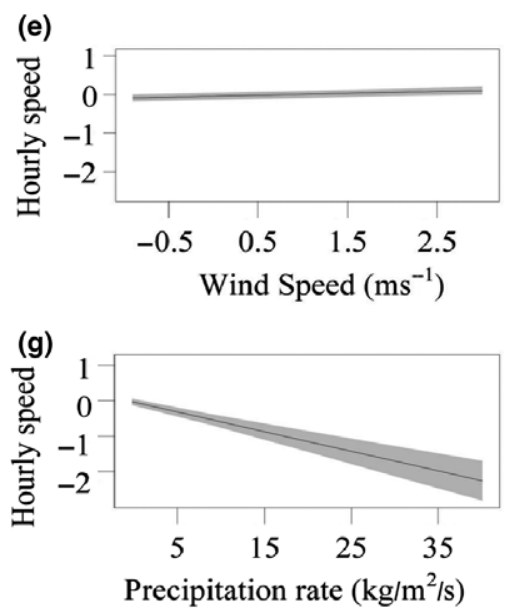

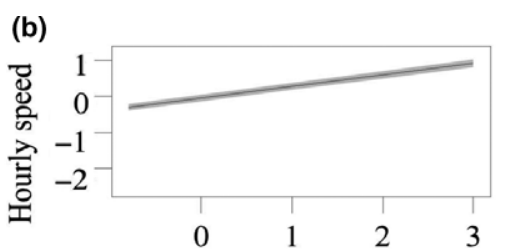

Planetary boundary layer height $(\mathrm{m})$
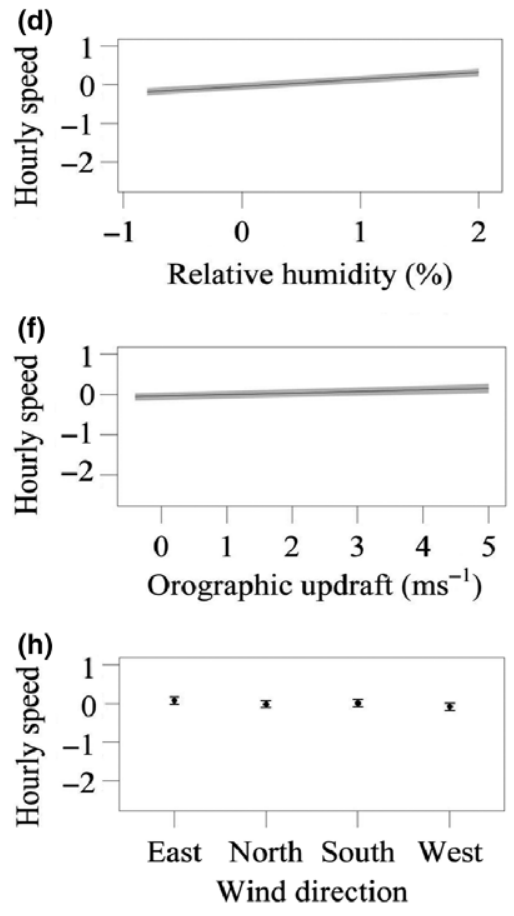

Figure 8. Model estimated values of hourly speed (log-transformed) of Golden Eagles in California, as predicted by meteorological variables (a) downward solar radiation, (b) planetary boundary layer height, (c) barometric pressure, (d) surface lifted index, (e) relative humidity, (f) wind speed, (g) precipitation rate and (h) wind direction. The plot uses estimated values from the top meteorological model predicting hourly speed (Table 1). The variables were rescaled for modelling purposes (see text). Grey bands represent $95 \%$ confidence intervals.

multiple meteorological variables including the height of the planetary boundary layer, precipitation rate and downward solar radiation. Finally, $\mathrm{BCR}$, hour of the day and month of the year were comparatively less influential, and effect estimates of many of these variables were not different from zero.

\section{DISCUSSION}

There are many different hypotheses to explain potential drivers of animal movement. Parsing out the relative importance of these hypotheses becomes even more difficult for birds that are dependent on conditions both on the ground and in the gaseous medium in which they move. Many previous studies have generally evaluated a single category of environmental variable (e.g. a suite of either meteorological or topographic variables) as potential drivers of movement response (e.g. Katzner et al. 2012, Duerr et al. 2015, Harel et al. 2016, Pirotta et al. 2018, Poessel et al. 2018). Our analyses suggest that focusing on a single category of environmental variable, usually weather, as a determinant of movement would have oversimplified understanding of this system. Instead, we saw that many of the processes or parameters we described function together to determine movement behaviour and that weather variables were unexpectedly less influential than were 
Table 4. Results of comparison of combinations of the top models from Table 3, describing factors affecting hourly speeds of Golden Eagles in California, 2012-2016.

\begin{tabular}{lccc}
\hline Hourly speed models & AICc & $\Delta$ AIC & $w_{\mathrm{i}}$ \\
\hline $\begin{array}{l}\text { Topo + Met + Hour } \\
\text { + Demo }\end{array}$ & 668785.30 & 0.00 & $\geq 0.99$ \\
Topo + Hour + Demo & 670102.90 & 1317.6 & 0.00 \\
Topo + Met + Hour & 670121.02 & 1335.72 & 0.00 \\
Topo + Met + Demo & 670268.05 & 1482.75 & 0.00 \\
Topo + Met & 674653.24 & 5867.94 & 0.00 \\
Met + Hour + Demo & 682511.72 & 13726.4 & 0.00 \\
Met + Demo & 684063.80 & 15278.5 & 0.00 \\
Topo + Demo & 684365.52 & 15580.2 & 0.00 \\
Met + Hour & 683601.26 & 14816 & 0.00 \\
Met & 688302.74 & 19517.4 & 0.00 \\
Topo + Hour & 671263.24 & 2477.94 & 0.00 \\
Topo & 685622.87 & 16837.6 & 0.00 \\
Hour + Demo & 684623.49 & 15838.2 & 0.00 \\
Demo & 700059.57 & 31274.3 & 0.00 \\
Hour & 685872.62 & 17087.3 & 0.00 \\
\hline
\end{tabular}

We used linear mixed effects with log-transformed hourly speeds of Eagles as the response variable, a combination of demographic (Demo), diel (Hour), topographic (Topo) and meteorological (Met) variables from our top models in Table 3 as fixed effects, and bird ID and year as random effects. We also added a lagged-response covariate as a fixed effect. We used ascending $\triangle \mathrm{AIC}$ to rank the models

topographic variables. Identifying these key covariates of movement, and their interactions, allowed us to improve our understanding and gain new insights into the potential internal and external drivers of movement. Finally, by comparing parameters that differentially influenced the two distinct response variables we considered, we also gained fresh insights into the internal competition and fundamental trade-offs animals face when making decisions about when and how to move.

\section{Internal state}

The internal state of an animal can influence its movement in many ways. Hunger, reproductive status, age and many other factors can all determine movement decisions (Nathan et al. 2008). We saw strong evidence that an individual Eagle's reproductive potential influenced its movement. Although such evidence is expected by theory describing species that engage in territorial defence and parental care, such a movement response is difficult to measure and thus has rarely, if ever, been documented. Golden Eagles in California start defending their territory as early as October (Braham et al. 2015), and although the nesting cycle in the southern part of the state is earlier than that farther north, birds in both regions generally are tending nests from January to July. Our analyses indicated that both male and female Eagles were less likely to be in motion (i.e. they decreased their activity) as the nesting season progressed (Fig. 2c). Even though we did not have information on breeding success, we saw that this was especially true for females that, when nesting, incubate eggs and brood nestlings for a greater proportion of time than do males. That said, year-round, males were, on average, more likely to be in motion than were females (Fig. 2c).

We can draw several biological conclusions from these sex-specific movement patterns. First, they probably reflect seasonal changes in nesting biology. Regardless of breeding status, the behaviours most likely to decrease as the nesting season progresses are territory defence and nest building. Both of these are energy-intensive behaviours that are most prevalent during the period when Eagles were most likely to be in motion - the earliest part of the nesting cycle. In addition, our analyses showed that Eagles were more likely to be in motion in spring and autumn, the periods when some of them make longer distance non-breeding movements. Secondly, and perhaps less intuitively, it seems that males, in general, move more than females. Two broadly applicable mechanisms can explain this pattern. First, during the nesting season when females are gravid or involved in stationary incubation behaviour, males are often engaged in movement-intensive foraging or territory defence behaviours. Secondly, these data also could mean that, year-round, males may spend a greater proportion of their time defending a territory. Although each movement behaviour may have unique energetic costs (territorial displays are often low-energy soaring, whereas hunting can involve more energy-intensive soaring or flapping), it is nevertheless true that when a bird needs to be in motion more at one time of the year than other times, that time of the year is likely to be relatively more energetically expensive.

These interpretations explain the state-specific behaviour of territorial adult Golden Eagles, but they need further development to incorporate behaviour of non-territorial birds. The age-related differences we observed in the probability of being in motion provide insight into how internal state, 
manifested in territoriality, reproductive behaviour and experience, probably influences animal movement. Sub-adult birds, which are only rarely territorial, were more likely to be in motion and, when in motion, moved more quickly than adult birds. This is consistent with these birds ranging more widely than do territorial birds (a pattern that is observed elsewhere; Miller et al. 2016). Likewise, there is evidence that younger, non-territorial birds of many species are more likely to make migratory or pseudo-migratory movements even when they are from putatively 'sedentary' populations (Bloom et al. 2015). Such migratory movements involve being in motion and are often faster than local movements (Wheat et al. 2017). Finally, age-related differences in movement behaviour could also be indicative of a potential role of experience. In particular, there is good evidence from studies of migration that adults fly more efficiently than younger birds (Harel et al. 2016, Miller et al. 2016, Rus et al. 2017). Such efficiency can be from in-flight improvements or, as suggested by this work, by changes to the amount of time birds spend in flight. Thus, exploring these patterns in our data and their ecological significance helps our understanding of how internal state serves as a potential driver for Golden Eagle movement.

These state-related drivers of movement provide important and broadly generalizable insight into ecological and evolutionary processes. For example, we generally assume that foraging is the most energetically demanding part of an animal's life cycle (Shepard et al. 2011). However, we observed dramatic seasonal fluctuations in the probability of being in motion and a decline in adult movement as nestlings aged. This is counterintuitive, as this is the time period when nestling food needs, and often prey availability, may be at their maximum. These patterns suggest that territory defence may involve more movement, and thus be more energetically taxing, than is providing food for offspring. This finding is unexpected, as there is substantial evidence that energetic constraints limit productivity (e.g. supplemental feeding of offspring increases reproductive output; Ferrer et al. 2017). As such, our observations have substantial implications for our understanding of the causes and consequences of the energetic limitations for monogamous territorial species in general.

Likewise, the greater frequency of movements by younger birds also provides insight into potentially important demographic processes. For example, it is well established that young of most species experience higher mortality rates than adults (Gotelli 1998). Our data suggest that one of the reasons for this may be the greater risk and energetic demands associated with increased movement of younger birds. For example, mortality rates for Black Kites Milvus migrans peak between the first and second year of life (Sergio et al. 2011). Likewise, the benefits to an adult of finding a territory may be not only because it increases reproductive potential, but also, and perhaps counterintuitively, because holding a territory decreases requirements for movement and thus increases survivorship.

\section{External environment}

We also saw strong evidence that the external environment influenced Eagle movement. In fact, because this response was so substantial and because so many variables were relevant to this response, these patterns illustrate the complexity of the dependence of movement on the environmental state and the relative insignificance of weather to flight behaviour.

Most work on the movement of soaring birds points to the substantial importance of weather in determining flight behaviour (Duerr et al. 2015, Vansteelant et al. 2015, Rus et al. 2017, Poessel et al. 2018). It was therefore remarkable that this dataset suggests that when topography and weather are both considered, topographic features more strongly influence flight behaviour compared with weather variables. The difference between our work and prior work may be that in many cases, prior analysis has focused on a very few weather variables (e.g. two variables, tailwind and turbulent kinetic energy in Harel et al. 2016) and few, if any, topographic parameters (Panuccio et al. 2016, Poessel et al. 2018).

Our broadened focus illustrated that Eagle movement responses to variation in both topography and weather often were unexpectedly strong. In particular, northness of a slope (Fig. 3a) and increasing levels of orographic updraft (Fig. 4f), downward solar radiation (Fig. 4a) and the height of the planetary boundary layer (Fig. 4b) were all tightly correlated to the probability of an Eagle being in motion. Similar (although not identical) variables were strongly related to hourly speed (Figs 7 and 8). The unexpectedly narrow 
confidence intervals around many of these estimates are informative because they suggest the strong dependence of Eagle behaviour on these parameters that alter the medium in which they travel. They also suggest that Eagles are using updraft resources in direct response to their availability, an implication consistent with regular switching from one subsidy type to another (Katzner et al. 2015). Other species such as California Condors Gymnogyps californianus, which are even less well suited to flapping flight, show similar dependence, but to a substantially smaller suite of environmental variables (Poessel et al. 2018). These comparisons suggest the testable hypotheses that species that are well adapted to flapping flight respond to a relatively wider range of environmental variables, but with relatively weaker dependence on any single variable compared with eagles and condors.

Beyond weather and topography, the movement responses we measured also varied across the day and by ecoregions. Such responses contribute to our understanding of the strong interdependence of environmental and state-based potential drivers of flight behaviour. Diel cycles in behaviour, for example, probably reflect both diel cycles in the environment (i.e. thermal updraft strength generally peaks in the middle of the day, probably driving the movement peak we observed; Fig. a,b) and diel cycles in animal state (i.e. late in the day, Eagles may feel pressure to find a safe roost or to feed before roosting; Fig. a,b). Likewise, differences in behaviour in the five BCRs we considered were a reflection of the external and internal factors that affect the population of Eagles that reside in these ecoregions. Therefore, the variation in behaviour we observed is also a reflection of both spatial variation in the environment (i.e. desert climates are hotter, resulting in greater thermal potential, probably explaining the fast movement speeds in the desert BCRs; Fig. 6c) and spatial variation in internal state (i.e. earlier nesting in deserts means less movement earlier in the year; potential migration in the Great Basin BCR, less presumptive foraging effort, and less movement in areas of the Sierra Nevada BCR with abundant colonial ground squirrels; Fig. 6c). Although temporal patterns in movement have been relatively well studied (Omland \& Hoffman 1996, Soutullo et al. 2005, Cadahía et al. 2007, Poessel et al. 2016), the population-level spatial variation we measured in movement is not commonly incorporated into those studies. In fact, accounting for spatial variation in movement was central to accurate interpretation of our data and may be an important component to future work in this field.

\section{Relative significance of different types of variables}

By comparing the combinations of variables that influenced the two response variables we considered, we also gained new insight into the relative influence of different variable types when making decisions about when and how to move. Interestingly, the combination of topographic and meteorological variables that most influenced the decision to move generally were also those that affected hourly speed. For example, the variables that had the greatest influence on both processes were mostly those that supported the development of updrafts (topographic features such as aspect, roughness and, to a lesser degree, meteorological factors). The relatively greater importance of topography suggests that these soaring birds use topographically generated updrafts even when meteorological conditions were not optimal for generation of thermal updrafts. Together, these patterns suggest that movement responses are, to a degree, consistent, suggesting that animals decide to move for the same reasons they may be able to move optimally.

\section{CONCLUSION}

An individual's movement behaviour results from the dynamic interaction of four factors: the animal's capacity to navigate, its capacity to move, its internal state and the external environment (Nathan et al. 2008). In this study, the capacity to navigate and to move were, for the purposes of the data we considered here, essentially invariant. However, the internal state of the animals and the external environment they experienced were highly variable. The models we used to describe an animal's movement response to its internal state were, to a large degree, straightforward and interpretable, with only a few variables (age, sex, location, time of year) and interactions. In contrast, the models we used to describe an animal's movement response to two different types of environmental variation were far more complex.

Our analyses not only show how connections between both of these variable types together 
influence animal movement but also provide hints into the fitness value of different movement strategies, and they generate testable hypotheses for future study with a broader range of taxa. It is well established that animals must balance constraints of age, experience and reproduction. However, by evaluating these effects in the context of movement, we generated potential theories explaining energetic constraints on territorial animals (i.e. territorial defence may be more energetically demanding than provisioning young), the benefits of territoriality (i.e. improved survival of adults over sub-adults may be driven in part by lower risk and energetic expenditure resulting from moving less) and how flight physiology may impact response to weather (i.e. that adaption to flapping flight influences the degree to which flight behaviour responds to environmental variation). Our work also demonstrated that topographic influences are often more relevant than meteorological influences in determining patterns in flight behaviour. These hypotheses thus form a possible framework for further refinement of our understanding of the ecology of soaring birds and animal movement.

We thank Cellular Tracking Technologies, which manufactured the GPS-GSM transmitters used in this project. J. Shamoun-Baranes, Scott LaPoint, and a number of other reviewers and journal editors provided helpful comments to improve the manuscript. A large number of field technicians worked to trap, telemeter and sometimes recover the Eagles studied in this project. D.A.B and T.E.K. would like to thank Daniel Driscoll, Joe DiDonato and Brian Latta for field assistance, trapping and transmitter attachment, Eric Folmer for tree climbing assistance, Harvey Wilson and volunteers with the Golden Eagle Monitoring Team of the East Bay Regional Park District for field assistance, and the Gordon and Betty Moore Foundation, East Contra Costa County Habitat Conservancy, Contra Costa Water District, Next Era Energy, Inc., and the East Bay Regional Park District for funding. Access to the Tollhouse and Caliente Ranches, and Santa Rosa Plateau Reserve was provided by The Nature Conservancy. The Cattani Ranch was kindly made available by the Cattani family. The City of San Diego provided access to their natural area properties. Any use of trade, product, or firm names is for descriptive purposes only and does not imply endorsement by the U.S. Government.

\section{FUNDING STATEMENT}

This work was supported by the California Department of Fish and Wildlife (CDFW agreements P1182024, P1480006 and P1550004), San Diego Associations of Government, William J Toulis Trust, U.S. Fish and Wildlife Service, and the Bureau of Land Management (U.S. BLM contract L11PX02237) as well as the authors' institutions.

\section{ETHICS STATEMENT}

This research was conducted under IACUC approval at West Virginia University (protocol number 1603001797) and complies with the Guidelines to the Use of Wild Birds in Research. Trapping was conducted under a number of different California State and U.S. Federal bird banding permits; numbers and copies of permits are available upon request. At the time some of the telemetry systems were deployed, T.E.K. was a coowner of Cellular Tracking Technologies, the company that manufactured the telemetry units used in this study.

\section{AUTHOR CONTRIBUTIONS}

All authors contributed to the study design. T.E.K., D.A.B. and R.N.F. conceived the idea, design, experiment and raised funding. P.H.B., D.A.B. and T.E.K. led or organized trapping and telemetering of Eagles. M.S., T.E.K. and A.E.D. designed the research questions and statistical analyses and M.S. analysed the data. M.S. and T.E.K. led writing of the manuscript and all authors revised the manuscript.

\section{DATA AVAILABILITY STATEMENT}

All government-owned data have been placed in a publicly accessible USGS data archive.

\section{REFERENCES}

Anderson, D.R. 2007. Model Based Inference in the Life Sciences: A Primer on Evidence. New York, NY: Springer Science \& Business Media.

Anderson, D.R. \& Burnham, K.P. 2002. Avoiding pitfalls when using information-theoretic methods. J. Wildl. Manag. 66: 912-918.

Barton, K. 2019. MuMIn: Multi-Model Inference. R package version 1.43.6. 
Bloom, P.H. \& Clark, W.S. 2001. Molt and sequence of plumages of Golden Eagles and a technique for in-hand ageing. North Am. Bird Bander 26: 97-116.

Bloom, P.H., Clark, W.S. \& Kidd, J.W. 2007. Capture techniques. In Bildstein, K.L. \& Bird, D.M. (eds) Raptor Research and Management Techniques: 193-219. Blaine, MN: Hancock House Publishers.

Bloom, P.H., McCrary, M.D., Scott, J.M., Papp, J.M., Sernka, K.J., Thomas, S.E., Kidd, J.W., Henckel, E.H., Henckel, J.L. \& Gibson, M.J. 2015. Northward summer migration of Red-tailed Hawks fledged from southern latitudes. J. Raptor Res. 49: 1-17.

Bortolotti, G.R. 1984. Age and sex size variation in golden eagles. J. Field Ornithol. 55: 54-66.

Braham, M., Miller, T., Duerr, A.E., Lanzone, M., Fesnock, A., LaPre, L., Driscoll, D. \& Katzner, T. 2015. Home in the heat: dramatic seasonal variation in home range of desert golden eagles informs management for renewable energy development. Biol. Cons. 186: 225-232.

Cadahía, L., Urios, V. \& Negro, J.J. 2007. Bonelli's Eagle Hieraaetus fasciatus juvenile dispersal: hourly and daily movements tracked by GPS. Bird Study 54: 271-274.

Clobert, J., Galliard, L., Cote, J., Meylan, S. \& Massot, M. 2009. Informed dispersal, heterogeneity in animal dispersal syndromes and the dynamics of spatially structured populations. Ecol. Lett. 12: 197-209.

D'Eon, R.G. \& Delparte, D. 2005. Effects of radio-collar position and orientation on GPS radio-collar performance, and the implications of PDOP in data screening. J. Appl. Ecol. 42: 383-388.

Diehl, R.H. 2013. The airspace is habitat. Trends Ecol. Evol. 28: 377-379.

Dilts, T. 2015. Topography tools for ArcGIS. Available at: http://www.arcgis.com/home/item.html?xml:id=b13b3b40fa3c 43d4a23a1a09c5fe96b9 (accessed 12 May 2017).

Dodge, S., Bohrer, G., Weinzierl, R., Davidson, S.C., Kays, R., Douglas, D., Cruz, S., Han, J., Brandes, D. \& Wikelski, M. 2013. The environmental-data automated track annotation (Env-DATA) system: linking animal tracks with environmental data. Mov. Ecol. 1: 3.

Doherty, P.F., White, G.C. \& Burnham, K.P. 2012. Comparison of model building and selection strategies. J. Ornithol. 152: 317-323.

Doyle, J.M., Katzner, T.E., Bloom, P.H., Ji, Y., Wijayawardena, B.K. \& DeWoody, J.A. 2014. The genome sequence of a widespread apex predator, the Golden Eagle (Aquila chrysaetos). PLoS One 9: e95599.

Duerr, A.E., Miller, T.A., Lanzone, M., Brandes, D., Cooper, J., O'Malley, K., Maisonneuve, C., Tremblay, J.A. \& Katzner, T. 2015. Flight response of slope-soaring birds to seasonal variation in thermal generation. Funct. Ecol. 29: 779-790.

Dunstan, T. 1972. Radio-tagging Falconiform and Strigiform birds. Raptor Res. 6: 93-102.

Edwards, T.C. \& Kochert, M.N. 1986. Use of body weight and length of footpad as predictors of sex in Golden Eagles. J. Field Ornithol. 57: 317-319.

Evans, J.S., Oakleaf, J., Cushman, S.A. \& Theobald, D. 2014. An ArcGIS toolbox for surface gradient and geomorphometric modeling, Version 2.0.0.

Ferrer, M., Morandini, V., Baguena, G. \& Newton, I. 2017. Reintroducing endangered raptors: a case study of supplementary feeding and removal of nestlings from wild populations. J. Appl. Ecol. 55: 1360-1367.

Fieberg, J. \& Ditmer, M. 2012. Understanding the causes and consequences of animal movement: a cautionary note on fitting and interpreting regression models with timedependent covariates. Methods Ecol. Evol. 3: 983-991.

Fletcher, D., MacKenzie, D. \& Villouta, E. 2005. Modelling skewed data with many zeros: a simple approach combining ordinary and logistic regression. Environ. Ecol. Stat. 12: 4554.

Fox, J. \& Monette, G. 1992. Generalized collinearity diagnostics. J. Am. Stat. Assoc. 87: 178-183.

Gelman, A. 2008. Scaling regression inputs by dividing by two standard deviations. Stat. Med. 27: 2865-2873.

Giesbrecht, F.G. \& Burns, J.C. 1985. Two-stage analysis based on a mixed model: large-sample asymptotic theory and small-sample simulation results. Biometrics 41: 477486.

Gotelli, N.J. 1998. A Primer of Ecology, 2nd edn. Sunderland, MA: Sinauer Associates.

Graf, P.M., Mayer, M., Zedrosser, A., Hackländer, K. \& Rosell, F. 2016. Territory size and age explain movement patterns in the Eurasian beaver. Mamm. Biol. 81: 587-594.

Halsey, L.G. 2016. Terrestrial movement energetics: current knowledge and its application to the optimising animal. $J$. Exp. Biol. 219: 1424-1431.

Harel, R., Horvitz, N. \& Nathan, R. 2016. Adult vultures outperform juveniles in challenging thermal soaring conditions. Sci. Rep. 6: 27865.

Hays, G.C., Ferreira, L.C., Sequeira, A.M., Meekan, M.G., Duarte, C.M., Bailey, H., Bailleul, F., Bowen, W.D., Caley, M.J., Costa, D.P. \& Eguíluz, V.M. 2016. Key questions in marine megafauna movement ecology. Trends Ecol. Evol. 31: 463-475.

Jenness, J., Brost, B. \& Beier, P. 2013. Land Facet Corridor Designer: Extension for ArcGIS, v.1.2.884. Flagstaff, AZ: Jenness Enterprises.

Jollie, M. 1947. Plumage changes in the Golden Eagle. Auk 64: 549-576.

Katzner, T.E., Brandes, D., Miller, T., Lanzone, M., Maisonneuve, C., Tremblay, J.A., Mulvihill, R. \& Merovich, G.T. 2012. Topography drives migratory flight altitude of Golden Eagles: implications for on-shore wind energy development. J. Appl. Ecol. 49: 1178-1186.

Katzner, T.E., Turk, P.J., Duerr, A.E., Miller, T.A., Lanzone, M.J., Cooper, J.L., Brandes, D., Tremblay, J.A. \& Lemaitre, J. 2015. Use of multiple modes of flight subsidy by a soaring terrestrial bird, the Golden Eagle Aquila chrysaetos, when on migration. J. R. Soc. Interface 12: 20150530.

Kenward, R.E. 1985. Raptor Radio-Tracking and Telemetry. ICBP Technical Publication 5: 409-420.

Kuznetsova, A., Brockhoff, P.B. \& Christensen, R.H.B. 2017. ImerTest Package: tests in linear mixed effects models. J. Stat. Softw. 82: 1-26.

Mainwaring, M.C. \& Hartley, I.R. 2013. The energetic costs of nest building in birds. Avian Biol. Res. 6: 12-17.

Miller, T.A., Brooks, R.P., Lanzone, M.J., Brandes, D., Cooper, J., Tremblay, J.A., Wilhelm, J., Duerr, A. \& Katzner, T.E. 2016. Limitations and mechanisms influencing the migratory performance of soaring birds. Ibis 158: 116134. 
Miller, T.A., Brooks, R.P., Lanzone, M.J., Cooper, J., O'Malley, K., Brandes, D., Duerr, A. \& Katzner, T.E. 2017. Summer and winter space use and home range characteristics of Golden Eagles (Aquila chrysaetos) in eastern North America. Condor 119: 697-719.

Nagy, M., Couzin, I.D., Fiedler, W., Wikelski, M. \& Flack, A. 2018. Synchronization, coordination and collective sensing during thermalling flight of freely migrating White Storks. Philos. T. R. Soc. B Biol. Sci. 373: 20170011.

Nathan, R., Getz, W.M., Revilla, E., Holyoak, M., Kadmon, R., Saltz, D. \& Smouse, P.E. 2008. A movement ecology paradigm for unifying organismal movement research. Proc. Natl Acad. Sci. USA 105: 19052-19059.

Omland, K. \& Hoffman, S. 1996. Seasonal, diel, and spatial dispersion patterns of Golden Eagle autumn migration in Southwestern Montana. Condor 98: 633-636.

Panuccio, M., Stanzione, V., Catoni, C., Santini, M. \& Dell'Omo, G. 2016. Radar tracking reveals influence of crosswinds and topography on migratory behaviour of European honey buzzards. J. Ethol. 34: 73-77.

Pinheiro, J., Bates, D., DebRoy, S. \& Sarkar, D. 2015. R Development Core Team. NIme: Linear and Nonlinear Mixed Effects Models. R Package Version: 3.1-109.

Pirotta, E., Katzner, T., Miller, T.A., Duerr, A.E., Braham, M.A. \& New, L. 2018. State-space modelling of the flight behaviour of a soaring bird provides new insights to migratory strategies. Funct. Ecol. 32: 2205-2215.

Poessel, S.A., Bloom, P.H., Braham, M.A. \& Katzner, T.E. 2016. Age- and season-specific variation in local and longdistance movement behaviour of Golden Eagles. Eur. J. Wildl. Res. 62: 377-393.

Poessel, S.A., Brandt, J., Miller, T.A. \& Katzner, T.E. 2018. Meteorological and environmental variables affect flight behaviour and decision-making of an obligate soaring bird, the California Condor Gymnogyps californianus. Ibis 160: 36-53.

R Core Team 2012. R: A Language and Environment for Statistical Computing. Vienna, Austria: R Foundation for Statistical Computing.

Riley, S.J., DeGloria, S.D. \& Elliot, R. 1999. A terrain ruggedness index that quantifies topographic heterogeneity. Intermt. J. Sci. 5: 23-27.

Roberts, D.W. 1986. Ordination on the basis of fuzzy set theory. Vegetatio 66: 123-131.

Rus, A.I., Duerr, A.E., Miller, T.A., Belthoff, J.R. \& Katzner, T.E. 2017. Counterintuitive roles of experience and weather on migratory performance. Auk 134: 485-497.

Sapir, N., Wikelski, M., Avissar, R. \& Nathan, R. 2011. Timing and flight mode of departure in migrating European bee-eaters in relation to multi-scale meteorological processes. Behav. Ecol. Sociobiol. 65: 1353-1365.

Sergio, F. \& Newton, I. 2003. Occupancy as a measure of territory quality. J. Anim. Ecol. 72: 857-865.

Sergio, F., Tavecchia, G., Blas, J., Lopez, L., Tanferna, A. \& Hiraldo, F. 2011. Variation in age-structured vital rates of a long-lived raptor: implications for population growth. Basic Appl. Ecol. 12: 107-115.

Shamoun-Baranes, J., Bouten, W., van Loon, E.E., Meijer, C. \& Camphuysen, C.J. 2016. Flap or soar? How a flight generalist responds to its aerial environment. Philos $T R$ Soc B. 371: 20150395.
Shepard, E.L., Lambertucci, S.A., Vallmitjana, D. \& Wilson, R.P. 2011. Energy beyond food: foraging theory informs time spent in thermals by a large soaring bird. PLoS One 6: e27375.

Smith, T.M. \& Shugart, H.H. 1987. Territory size variation in the Ovenbird: the role of habitat structure. Ecology 68: 695-704.

Soutullo, A., Urios, V. \& Ferrer, M.D. 2005. How far away in an hour? Daily movements of juvenile Golden Eagles (Aquila chrysaetos) tracked with satellite telemetry. J. Ornithol. 147: 69-72.

U.S. Geological Survey (USGS) 2015. The National Map, 3D Elevation Program website. Available at: http://nationalma p.gov/3dep_prodserv.html.

U.S. NABCI Committee 2000. North American Bird Conservation Initiative: Bird conservation region descriptions, a supplement to the North American Bird Conservation Initiative Bird Conservation Regions Map.

Van Cleave, E.K., Bidner, L.R., Ford, A.T., Caillaud, D., Wilmers, C.C. \& Isbell, L.A. 2018. Diel patterns of movement activity and habitat use by leopards (Panthera pardus pardus) living in a human-dominated landscape in central Kenya. Biol. Cons. 226: 224-237.

Vansteelant, W.M.G., Bouten, W., Klaassen, R.H.G., Koks, B.J., Schlaich, A.E., van Diermen, J., Van Loon, E.E. \& Shamoun-Baranes, J. 2015. Regional and seasonal flight speeds of soaring migrants and the role of weather conditions at hourly and daily scales. J. Avian Biol. 46: 2539.

Vansteelant, W.M., Shamoun-Baranes, J., Manen, W., Diermen, J. \& Bouten, W. 2017. Seasonal detours by soaring migrants shaped by wind regimes along the East Atlantic Flyway. J. Anim. Ecol. 86: 179-191.

Watson, J. 2010. The Golden Eagle. London: T. and A.D. Poyser.

Weimerskirch, H., Cherel, Y., Delord, K., Jaeger, A., Patrick, S.C. \& Riotte-Lambert, L. 2014. Lifetime foraging patterns of the wandering albatross: life on the move!. $J$. Exp Mar Biol Ecol. 450: 68-78.

Welsh, A.H., Cunningham, R.B., Donnelly, C.F. \& Lindenmayer, D.B. 1996. Modelling the abundance of rare species: statistical models for counts with extra zeros. Ecol. Model. 88: 297-308.

Wheat, R.E., Lewis, S.B., Wang, Y., Levi, T. \& Wilmers, C.C. 2017. To migrate, stay put, or wander? Varied movement strategies in Bald Eagles (Haliaeetus leucocephalus). Mov. Ecol. 5: 9.

Wikelski, M. \& Kays, R. 2016. Movebank: archive, analysis and sharing of animal movement data. Hosted by the Max Planck Institute for Ornithology. Available at: http:// www.movebank.org (accessed 1 September 2016).

Wittemyer, G., Polansky, L., Douglas-Hamilton, I. \& Getz, W.M. 2008. Disentangling the effects of forage, social rank, and risk on movement autocorrelation of elephants using Fourier and wavelet analyses. Proc. Natl Acad. Sci. USA 105: 19108-19113.

Zuur, A., leno, E.N., Walker, N.J., Saveliev, A.A. \& Smith, G.M. 2009. Mixed Effects Models and Extensions in Ecology With R. New York: Springer.

Zuur, A.F., leno, E.N. \& Elphick, C.S. 2010. A protocol for data exploration to avoid common statistical problems. Methods Ecol. Evol. 1: 3-14. 
Received 3 April 2019; revision accepted 19 July 2019. Associate Editor: Kurt Burnham.

\section{SUPPORTING INFORMATION}

Additional supporting information may be found online in the Supporting Information section at the end of the article.

Data S1. Description of Bird Conservation Regions.

Figure S1. Rationale for choosing a threshold for the classification of hourly speed of Eagles.

Table S1. Correlation matrix for variables used in the analysis.

Table S2. Multicollinearity test for covariates used in the generalized linear mixed models.

Table S3. Multicollinearity test for covariates used in the linear mixed effect models.

Table S4. Summary information of tracked Golden Eagles.

Table S5. Model averaging results from best performing generalized linear mixed effect model explaining the influence of meteorological variable on the probability of being in motion.

Table S6. Results of the model describing effect of hour on the decision to move.
Table S7. Results of the model describing effect of demographic, diel, topographic and meteorological factors on the decision to move.

Table S8. Analysis of deviance table for model describing the effect of demographic, topographic and meteorological covariates on the probability of movement.

Table S9. Model averaging results from the best performing linear mixed effect model explaining the influence of demographic variables on hourly speed.

Table S10. Model averaging results from the best performing linear mixed effect model explaining the influence of meteorological variables on hourly speed.

Table S11. Results of the model describing the effect of hour on hourly speed.

Table S12. Results of the model describing the effect of demographic, diel, topographic and meteorological factors on hourly speed.

Table S13. Type III analysis of variance table for the model describing the effect of demographic, topographic and meteorological covariates on hourly speed. 